\title{
Exact solution to surface displacement associated with sources distributed on an inclined plane
}

\author{
Arabinda Roy ${ }^{1}$ and Ajit $\mathrm{De}^{2^{*}}$ \\ ${ }^{1}$ Department of Applied Mathematics, Calcutta University, 92, A.P.C. Road, Kolkata-700009, INDIA. \\ ${ }^{2}$ Department of Mathematics, Siliguri College, Siliguri-734001, West Bengal, INDIA. \\ *Corresponding Author: e-mail: ajit_math@rediffmail.com
}

\begin{abstract}
Usually an exact solution to the surface displacement in an elastic half space is available for sources parallel to the surface. Here we consider a buried elliptic source distributed over an inclined plane. Circular and Point sources have been considered as particular cases of an elliptic source. Cagniard method has been used. We present some graphical plots of the surface displacement for various inclination of the fault plane.
\end{abstract}

Keywords: Cagniard Method, Elliptic Source, Inclined plane, Surface displacement.

\section{Introduction}

An earthquake is usually assumed to be generated by a buried source model in an elastic half-space. The most widely discussed finite source models are the circular disc or ring load models. Gakenheimer and Miklowitz (1969) used Cagniard-de-Hoop method to obtain the exact transient motion of the surface for both stationary or moving line and point sources. The complete solution to the circular source problem was proposed by Mitra (1964). Tupholme (1970) \& Roy (1975) also have studied the pulse shape near wave arrivals particularly at the outer and inner head waves using the Cagniard-de-Hoop method. Gridin (1999) studied the pulses in the region where different rays coming from the edge interact with direct waves. Zeng and Liang (2002) used Laplace-Hankel mixed transform and transfer matrix techniques along with the Fast Hankel transform algorithm for an impulsive ring source within a layered elastic media. Savage (1966) recommended an elliptic fault as an earthquake model. Roy(1981) studied the transient response of an elastic half-space due to a normal pressure on an elliptic area. The most of the earlier studies are limited to finite sources on the surface or parallel to the surface. However mostly earthquake sites are along some inclined geological faults. To model realistic earthquake source we have considered here a buried elliptic source along an inclined plane which experiences a normal pressure. The Circular source can be considered as a particular case of the elliptic source. The exact transient response due to the source is obtained by using Cagniard De-Hoop method with some graphical representations.

\section{Formulation of the Problem}

Let $(\mathrm{x}, \mathrm{y}, \mathrm{z})$ be an orthogonal Cartesian coordinate system with origin at the centre of the elliptic source and xy- plane parallel to the free surface $z=-h$ in an uniform elastic half-space. The $z$-axis is perpendicular to the free surface and directed inside the half space. Another coordinate system $\left(\zeta_{1}, \zeta_{2}, \zeta_{3}\right)$, called the source coordinate system, is introduced at the centre of the elliptic area such that the plane $\zeta_{3}=0$ is the inclined plane (Fig.1). The two coordinate systems (x,y,z) and $\left(\zeta_{1}, \zeta_{2}, \zeta_{3}\right)$ are related by 


$$
\left(\begin{array}{l}
x \\
y \\
z
\end{array}\right)=\bar{A}\left(\begin{array}{l}
\zeta_{1} \\
\zeta_{2} \\
\zeta_{3}
\end{array}\right)=\left(\begin{array}{lll}
\cos \lambda & -\sin \lambda & 0 \\
\sin \lambda \cos \delta & \cos \lambda \cos \delta & -\sin \delta \\
\sin \lambda \sin \delta & \cos \lambda \sin \delta & \cos \delta
\end{array}\right)\left(\begin{array}{l}
\zeta_{1} \\
\zeta_{2} \\
\zeta_{3}
\end{array}\right)
$$

where $\lambda$ and $\delta$ are respectively slip and dip angles of the inclined fault plane (Fig.1).

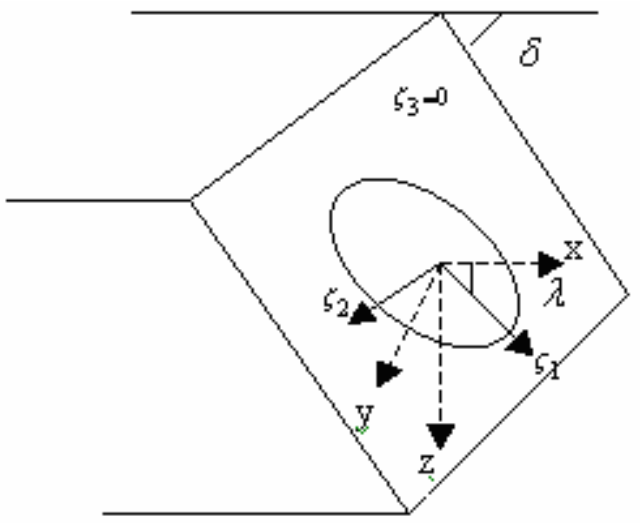

Figure 1. Elliptic source with two coordinate systems.

Let a constant normal impulsive pressure acts on an elliptic area along an inclined plane $\zeta_{3}=0$ at a depth 'h' below the surface of an elastic half-space. Thus the stress components on the fault plane $\zeta_{3}=0$ can be specified as

$$
\begin{aligned}
& \tau \zeta_{3} \zeta_{3}=-\mathrm{P} H\left(1-\sqrt{\frac{\zeta_{1}{ }^{2}}{a^{2}}+\frac{\zeta_{2}{ }^{2}}{b^{2}}}\right) \delta(t) \delta\left(\zeta_{3}\right) \\
& \tau \zeta_{3} \zeta_{1}=0=\tau \zeta_{3} \zeta_{2}
\end{aligned}
$$

where $\mathrm{P}$ is a constant, $\mathrm{H}(\mathrm{x})$ and $\delta(\mathrm{t})$ are respectively Heaviside's and Dirac's delta functions \& $\mathrm{a}$ and $\mathrm{b}$ are the semi-axes of the elliptic area. In a particular case, for a Circular source (i.e., $\mathrm{a}=\mathrm{b}$ ) the equation (2.2) can be expressed as

$$
\begin{aligned}
& \tau \zeta_{3} \zeta_{3}=-\mathrm{P} H\left(a-\sqrt{\zeta_{1}{ }^{2}+\zeta_{2}{ }^{2}}\right) \delta(t) \delta\left(\zeta_{3}\right) \\
& \tau \zeta_{3} \zeta_{1}=0=\tau \zeta_{3} \zeta_{2}
\end{aligned}
$$

The condition at the free surface is given by, $\quad \tau_{z x}=\tau_{z y}=\tau_{z z}=0 \quad$ on $\mathrm{z}=-\mathrm{h}$

Roy (1979) has shown the method of evaluation of the displacement field due to a stress distribution on an inclined fault plane in an integral form. Following Roy(1979), the transformed vertical surface displacement for the stress distribution (2.2) can be written as,

$$
u(p)=\frac{1}{2 \pi \mu}\left[\int_{-\infty}^{\infty} \int_{-\infty}^{\infty} \frac{\exp [i(\xi x+\eta y)]}{F(\xi, \eta)}\left\{A \exp \left(-\zeta_{\alpha} h\right)\left\{i\left(2 \xi^{2}+2 \eta^{2}+\left(p^{2} / \beta^{2}\right)\right)\right\}-B \exp \left(-\zeta_{\beta} h\right) 2 i \zeta_{\alpha}\right\}\right] d \xi d \eta
$$


where

$$
\begin{aligned}
& u(p)=\int_{0}^{\infty} u(t) \exp (-p t) d t \\
& F(\xi, \eta)=\left(2 \xi^{2}+2 \eta^{2}+\left(p^{2} / \beta^{2}\right)\right)^{2}-4\left(\xi^{2}+\eta^{2}\right) \zeta_{\alpha} \zeta_{\beta} \\
& A=P a b\left(\eta \sin \delta+i \zeta_{\alpha} \cos \delta\right) \frac{J_{1}\left(\sqrt{a^{2} k_{\alpha}{ }^{2}+b^{2} v_{\alpha}{ }^{2}}\right)}{\sqrt{a^{2} k_{\alpha}{ }^{2}+b^{2} v_{\alpha}{ }^{2}}} \\
& B=P a b\left(\eta \sin \delta \zeta_{\beta}+i\left(\xi^{2}+\eta^{2}\right) \cos \delta\right) \frac{J_{1}\left(\sqrt{a^{2} k_{\beta}{ }^{2}+b^{2} v_{\beta}{ }^{2}}\right)}{\sqrt{a^{2} k_{\beta}{ }^{2}+b^{2} v_{\beta}{ }^{2}}} \\
& k_{\alpha}=\cos \lambda \xi+\sin \lambda \cos \delta \eta-i \zeta_{\alpha} \sin \lambda \sin \delta, v_{\alpha}=-\sin \lambda \xi+\cos \lambda \cos \delta \eta-i \zeta_{\alpha} \cos \lambda \sin \delta \\
& k_{\beta}=\cos \lambda \xi+\sin \lambda \cos \delta \eta-i \zeta_{\beta} \sin \lambda \sin \delta, v_{\beta}=-\sin \lambda \xi+\cos \lambda \cos \delta \eta-i \zeta_{\beta} \cos \lambda \sin \delta \\
& \zeta_{\alpha}=\left(\xi^{2}+\eta^{2}+\left(p^{2} / \alpha^{2}\right)\right)^{1 / 2}, \zeta_{\beta}=\left(\xi^{2}+\eta^{2}+\left(p^{2} / \beta^{2}\right)\right)^{1 / 2}, i=\sqrt{-1}
\end{aligned}
$$

$\alpha=[(\lambda+2 \mu) / \sigma]^{1 / 2}$ and $\beta=(\mu / \sigma)^{1 / 2}$ are the $\mathrm{P}$ and $\mathrm{S}$ wave velocities of the elastic medium with $\lambda, \mu$ and $\sigma$ being the Lame's constants and density. In a particular case for $\mathrm{a}=\mathrm{b}$, the similar expressions for a Circular source can be easily written from (2.4) \& (2.5). In (2.4) the branch cuts are chosen such that $\operatorname{Re}\left(\zeta_{\alpha}\right)>0$ and $\operatorname{Re}\left(\zeta_{\beta}\right)>0$. It is to be noted that the vertical component of displacement for $\mathrm{SH}$-wave is absent and it exists only for horizontal component of the displacement field with $\delta \neq 0^{\circ}$. The terms A \& B depend on the particular source geometry.

\section{Evaluation of the Integral}

First P-wave is considered. To evaluate the integral using Cagniard-de-Hoop technique, the transformed vertical surface displacement associated with the $\mathrm{P}$ wave is written as

$$
u_{p}(p)=\int_{-\infty}^{\infty} \int_{-\infty}^{\infty} M_{p}(\xi, \eta) \frac{J_{1}\left[b \sqrt{\frac{a^{2} b^{2} k_{\alpha}{ }^{2}+v_{\alpha}{ }^{2}}{2}}\right.}{\sqrt{\frac{a^{2}}{b^{2}} k_{\alpha}{ }^{2}+v_{\alpha}{ }^{2}}} \exp \left[i(\xi x+\eta y)-\zeta_{\alpha} h\right] d \xi d \eta
$$

where $u_{p}(p)=\int_{0}^{\infty} u_{p}(t) \exp (-p t) d t$ and the complete expression for $M_{p}(\xi, \eta)$, containing term involving $\left(\xi^{2}+\eta^{2}+\left(p^{2} / \alpha^{2}\right)\right)^{1 / 2}$, can be easily written from (2.4). Now in (3.1), $\xi$ and $\eta$ are respectively replaced by $(p / \alpha) \xi$ and $(p / \alpha) \eta$. Thus (3.1) takes the form

$$
u_{p}(p)=\int_{-\infty}^{\infty} \int_{-\infty}^{\infty} M_{p}((p / \alpha) \xi,(p / \alpha) \eta) \frac{J_{1}\left[\left(\frac{p}{\alpha}\right) b \sqrt{\left.\frac{a^{2} b^{2} k_{P}{ }^{2}+v_{P}{ }^{2}}{}\right]}\right.}{\sqrt{\frac{a^{2}}{b^{2}} k_{P}{ }^{2}+v_{P}{ }^{2}}} \exp \left[\left(\frac{p}{\alpha}\right)\left\{i(\xi x+\eta y)-\zeta_{p} h\right\}\right] d \xi d \eta
$$

Where

$$
\eta_{p}=\eta \cos \delta-i \zeta_{p} \sin \delta, \zeta_{p}=\left(\xi^{2}+\eta^{2}+1\right)^{1 / 2}
$$

In (3.2) we make the change over from (x,y,z) to $\left(\zeta_{1}, \zeta_{2}, \zeta_{3}\right)$ by means of the transformation (2.1), i.e, the station (x, y,-h) on the free surface is now viewed in the coordinate system $\left(\zeta_{1}, \zeta_{2}, \zeta_{3}\right)$. Now using (3.3) and the integral representation of Bessel function of order one, (3.2) can be written as 
$u_{p}(p)=\int_{-\infty}^{\infty} \int_{-\infty}^{\infty} \int_{-\pi}^{\pi} \frac{N_{p}(q, \omega)}{\left(\frac{a^{2}}{b^{2}} \xi^{\prime 2}+\eta_{p}^{\prime 2}\right)} \exp \left[-\left(\frac{p}{\alpha}\right)\left\{-i q S+\zeta_{3}\left(q^{2}+\omega^{2}+1\right)^{1 / 2}\right\}\right] d \phi d q d \omega$

where

$N_{p}(q, \omega)=i \frac{M_{p}((p / \alpha) \xi,(p / \alpha) \eta)}{2 \pi\left(q^{2}+\omega^{2}+1\right)^{1 / 2}}\left(\frac{a}{b} \xi^{\prime} \cos \phi+\eta_{p}^{\prime} \sin \phi\right)\left(\xi^{2}+\eta^{2}+1\right)^{1 / 2}$

$\eta_{p}^{\prime}=\eta_{p} \cos \lambda-\xi \sin \lambda$

$\xi^{\prime}=\eta_{p} \sin \lambda+\xi \cos \lambda$

$\frac{a^{2}}{b^{2}} k_{P}^{2}+v_{P}^{2}=\eta_{p}^{\prime 2}+\frac{a^{2}}{b^{2}} \xi^{\prime 2}$

and the De-Hoop transformation

$\xi^{\prime}=q \cos \psi_{1}-\omega \sin \psi_{1}, \eta_{p}^{\prime}=q \sin \psi_{1}+\omega \cos \psi_{1}$

where

$\cos \psi_{1}=\frac{\zeta_{1}-a \cos \phi}{S}, \sin \psi_{1}=\frac{\zeta_{2}-b \sin \phi}{S}$

$S=\sqrt{\left(\zeta_{1}-a \cos \phi\right)^{2}+\left(\zeta_{2}-b \sin \phi\right)^{2}}$

The similar expressions for the circular source can be obtained by using $\mathrm{a}=\mathrm{b}$ in the above equations.

Now transferring the path of integration from the real q-axis to the Cagniard path, given by

$\alpha . t=-i q S+\zeta_{3}\left(q^{2}+\omega^{2}+1\right)^{1 / 2}$

and making use of the dimensionless variables $\tau=\frac{\beta . t}{\rho}, \zeta_{3}^{\prime}=\frac{\zeta_{3}}{\rho}, S^{\prime}=\frac{S}{\rho}$

where $\left.\rho=\left[S^{2}+\zeta_{3}{ }^{2}\right]^{1 / 2}=\left[\zeta_{1}-a \cos \phi\right)^{2}+\left(\zeta_{2}-b \sin \phi\right)^{2}+\zeta_{3}{ }^{2}\right]^{1 / 2}$

we have after taking inverse Laplace transform,

$u_{p}=u_{p 1}+u_{p 2}$

where $u_{p 1}$ is the contribution from the Cagniard's path and $u_{p 2}$ is the contribution from the pole at $\xi^{\prime}=i \frac{b}{a} \eta_{p}^{\prime}$. Now

$u_{p 1}=H\left(\tau-\frac{\beta}{\alpha}\right) \int_{-\pi}^{\pi} \int_{-\omega_{p}}^{\omega_{p}} \frac{N_{p}(q, \omega)}{\left(\frac{a^{2}}{b^{2}} \xi^{2}+\eta_{p}^{\prime 2}\right)} \frac{d q}{d t} d \omega d \phi$

$u_{p 2}=4 \pi \operatorname{Re} \int_{-\pi}^{\pi} H\left(\tau-\frac{\beta \zeta_{3}^{\prime}}{\alpha}\right) H\left(\frac{\beta a b}{\alpha \sqrt{a^{2} b^{2}-S^{\prime 2} D^{2}}}-\tau\right)\left[\frac{N_{p}(q, \omega)}{\eta_{p}^{\prime}} \sqrt{\frac{q^{2}+\omega^{2}+1}{\frac{\alpha^{2}}{\beta^{2}} \tau^{2}-\omega^{2}-1}}\right] \xi^{\prime}=i \frac{b}{a} \eta_{p}^{\prime} d \phi$

where $D=a^{2} \cos ^{2} \psi_{1}+b^{2} \sin ^{2} \psi_{1}$

For the circular source (i.e., for $\mathrm{a}=\mathrm{b}$ ), the equations similar to (3.11) \& (3.12) can be expressed as

$u_{p 1}=H\left(\tau-\frac{\beta}{\alpha}\right) \int_{-\pi}^{\pi} \int_{-\omega_{p}}^{\omega_{p}} \frac{N_{p}(q, \omega)}{\left(q^{2}+\omega^{2}\right)} \frac{d q}{d t} d \omega d \phi$ 
$u p 2=4 \pi \operatorname{Re} \int_{-\pi}^{\pi} H\left(\tau-\frac{\beta \zeta_{3}^{\prime}}{\alpha}\right) H\left(\frac{\beta}{\alpha \zeta_{3}^{\prime}}-\tau\right)\left[\frac{\alpha N_{p}(i \omega, \omega)}{\rho S^{\prime} \omega}\right] \underbrace{}_{\omega=\frac{\alpha \tau-\beta \zeta_{3}}{\beta . S^{\prime}}} d \phi$

The vertical component of the surface displacement associated with SV -wave can be evaluated similarly except that here one has to consider the branch cuts giving rise to head waves.

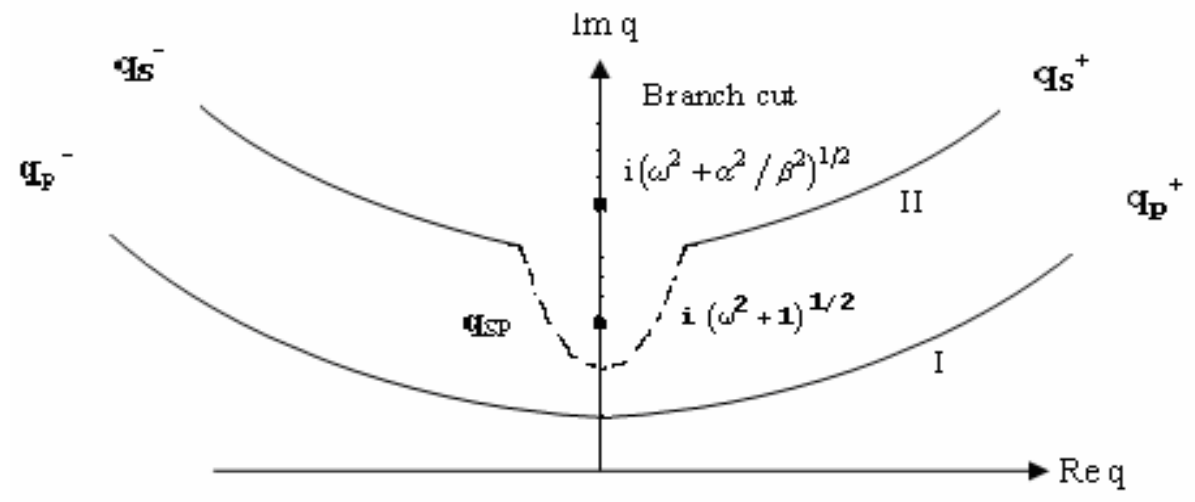

Figure 2. Cagniard paths corresponding to P-wave (I) and SV-wave (II) respectively. The dotted portion in the curve II represents the head wave contribution.

\section{Complete Expression for the Surface Displacement}

The complete expression for the vertical surface displacement $(\mathrm{u})$ for the stress distribution (2.2), can now be evaluated in a compact form by using the substitution $\omega^{2}=A_{1}{ }^{2}+\left(B_{1}{ }^{2}-A_{1}{ }^{2}\right) \sin ^{2} \psi$ where $A_{1}$ and $B_{1}$ are the upper and lower limits of integration in the expressions evaluated in section 3 . Thus

$$
u=u_{p}+u_{S V}+u_{S P}+u_{p}+u_{S V}
$$

where, for $\mathrm{j}=\mathrm{P}, \mathrm{SV}$

$$
\begin{aligned}
& u_{j}=H\left(\tau-\frac{\beta}{V_{j}}\right) \operatorname{Re} \int_{-\pi}^{\pi} \int_{-\pi / 2}^{\pi / 2}\left(\frac{\alpha}{\rho}\right) I_{j}\left(q_{j}^{+}, \omega_{j}\right) d \phi d \psi \\
& I_{j}\left(q_{j}^{+}, \omega_{j}\right)=2 N^{\prime}{ }_{j}\left(q_{j}^{+}, \omega_{j}\right)\left(q_{j}^{+2}+\omega_{j}{ }^{2}+d_{j}^{2}\right)^{1 / 2}, d_{j}=\left(\begin{array}{l}
1 \text { for } j=P \\
\frac{\alpha}{\beta} \text { for } j=S V^{.}
\end{array} V_{j}=\left(\begin{array}{l}
\alpha \text { for } j=P \\
\beta \text { for } j=S V
\end{array}\right.\right. \\
& \omega_{j}=\left(\left(\frac{\alpha^{2} \tau^{2}}{\beta^{2}}\right)-d_{j}{ }^{2}\right)^{1 / 2} \sin \psi, q_{j}^{+}=\frac{\alpha}{\beta}\left[i \tau \cdot S^{\prime}+\zeta_{3}{ }^{\prime}\left(\tau^{2}-\frac{\beta^{2}}{V_{j}{ }^{2}}\right)^{1 / 2} \cos \psi\right]
\end{aligned}
$$

The contributions due to the poles at $\xi^{\prime}=i \frac{b}{a} \eta_{p}^{\prime}$ and $\xi^{\prime}=i \frac{b}{a} \eta_{s}^{\prime}, u_{j}^{d}(j=P, S V)$ are obtained as

$$
u_{p}=4 \pi \operatorname{Re} \int_{-\pi}^{\pi} H\left(\tau-\frac{\beta \zeta_{3}^{\prime}}{V_{p}}\right) H\left(\frac{\beta a b}{\alpha \sqrt{a^{2} b^{2}-S^{\prime 2} D^{2}}}-\tau\right)\left[\frac{N_{P}(q, \omega)}{\eta_{p}^{\prime}} \sqrt{\frac{q^{2}+\omega^{2}+1}{\frac{\alpha^{2}}{\beta^{2}} \tau^{2}-\omega^{2}-1}}\right] \xi^{\prime}=i \frac{b}{a} \eta_{p}^{\prime} d \phi
$$




$$
u_{S V}^{d}=4 \pi \operatorname{Re} \int_{-\pi}^{\pi} H\left(\tau-\frac{\beta \zeta_{3}^{\prime}}{V_{S}}\right) H\left(\frac{\beta a b}{\alpha \sqrt{a^{2} b^{2}-S^{\prime 2} D^{2}}}-\tau\right)\left[\frac{N_{S V}(q, \omega)}{\eta_{s}^{\prime}} \sqrt{\frac{q^{2}+\omega^{2}+d_{S V}{ }^{2}}{\frac{\alpha^{2}}{\beta^{2}} \tau^{2}-\omega^{2}-d_{S V}{ }^{2}}}\right] \xi^{\prime}=i \frac{b}{a} \eta_{S}^{\prime} d \phi
$$

where

$$
N_{j}(q, \omega)=i \frac{M_{j}((p / \alpha) \xi,(p / \alpha) \eta)}{2 \pi\left(q^{2}+\omega^{2}+d_{j}{ }^{2}\right)^{1 / 2}}\left(\frac{a}{b} \xi^{\prime} \cos \phi+\eta_{p}^{\prime} \sin \phi\right)\left(\xi^{2}+\eta^{2}+d_{j}{ }^{2}\right)^{1 / 2}
$$

The head wave which is the contribution from the imaginary q-axis between $\left(0, i\left(\omega^{2}+\left(\frac{\alpha}{\beta}\right)^{2}\right)^{1 / 2}\right)$ and $\left(0, i\left(\omega^{2}+1\right)^{1 / 2}\right)$ is given by

$$
\begin{aligned}
& u S P=\operatorname{Re} \int_{-\pi}^{\pi} \int_{0}^{\pi / 2}\left(-\frac{2 . \alpha}{\rho}\right) H\left(\tau-t_{S P}^{\prime}\right) H(1-\tau) H\left(R^{\prime}-\frac{\beta}{\alpha}\right) \frac{i I_{S V}^{\prime}\left(q_{S P} \omega_{S P}\right) \cos \psi}{\left(\chi^{2}+\sin ^{2} \psi\right)^{1 / 2}} d \phi d \psi \\
& +\operatorname{Re} \int_{-\pi}^{\pi} \int_{0}^{\pi / 2}\left(-\frac{2 \alpha}{\rho}\right) H(\tau-1) H\left(t_{B}^{\prime}-\tau\right) H\left(R^{\prime}-\frac{\beta}{\alpha}\right) \frac{i \delta I^{\prime} S V\left(q_{S P}^{\prime}, \omega_{S P}^{\prime}\right) \cos \psi}{\left[\delta^{2} \sin ^{2} \psi+\left(\frac{R}{\rho}\right)^{2}\left(\tau^{2}-1\right)\right]^{1 / 2}} d \phi d \psi
\end{aligned}
$$

where

$$
\begin{aligned}
& I_{S V}^{\prime}(q, \omega)=\left[-\frac{i P a}{\pi} \frac{\left\{\eta \sin \delta \zeta_{S}+i\left(q^{2}+\omega^{2}\right) \cos \delta\right\}\left(2 i \zeta_{P}\right)}{2 \pi \mu \cdot F(q, \omega)}\right]\left[\frac{\left(\frac{a}{b} \xi^{\prime} \cos \phi+\eta_{s}^{\prime} \sin \phi\right)\left(q^{2}+\omega^{2}+\left(\frac{\alpha}{\beta}\right)^{2}\right)^{1 / 2}}{\left(\frac{a^{2}}{b^{2}} \xi^{\prime 2}+\eta_{s}^{\prime 2}\right)}\right] \\
& q_{S P}=\frac{i \alpha}{\beta}\left[\tau \cdot R^{\prime}-\left(h-z^{\prime}\right) \frac{\rho^{2}\left(m^{2} \sin ^{2} \psi+m^{2} \chi^{2}\right)^{1 / 2}}{\rho R^{\prime}}\right], \omega_{S P}=\left[\left\{\frac{\rho \alpha\left(\tau-t_{S P}^{\prime}\right)}{\beta \rho R^{\prime}}+1\right\}^{2}-1\right]^{1 / 2} \sin \psi \\
& q_{S P}^{\prime}=\frac{i \alpha \tau \cdot R^{\prime}}{\beta}-\frac{i \alpha}{\beta}\left(\frac{h-z^{\prime}}{\rho R^{\prime}}\right) \delta \sin \psi, \omega_{S P}^{\prime}=\left[T_{S}^{2}+\left(T_{S P}^{\prime}{ }^{2}-T_{S}{ }^{2}\right) \sin ^{2} \psi\right]^{1 / 2} \\
& T_{S}=\frac{\alpha}{\beta}\left(\tau^{2}-1\right)^{1 / 2}, T_{S P}^{\prime}=\left[\left\{\frac{\rho \alpha\left(\tau-t_{S P}^{\prime}\right)}{\beta \rho R^{\prime}}+1\right\}^{2}-1\right]^{1 / 2}, t_{S P}^{\prime}=\frac{\beta R^{\prime}}{\alpha}+\frac{\left(h-z^{\prime}\right)}{\rho}\left(1-\frac{\beta^{2}}{\alpha^{2}}\right)^{1 / 2} \\
& t_{B}^{\prime}=\frac{\rho}{\left(h-z^{\prime}\right)}\left(1-\frac{\beta^{2}}{\alpha^{2}}\right)^{1 / 2}, m \chi=R^{\prime}\left(1-\tau^{2}\right)^{1 / 2}, \delta=\left(1-\frac{\beta^{2}}{\alpha^{2}}\right)^{1 / 2}-\frac{\tau\left(h-z^{\prime}\right)}{\rho}
\end{aligned}
$$

The dimensionless variables $\tau$ and $R^{\prime} \quad$ are defined as $\quad \tau=\frac{\beta . t}{\rho}, R^{\prime}=\frac{R}{\rho} \quad$ where $\rho=\left(R^{2}+\left(h-z^{\prime}\right)^{2}\right)^{1 / 2}, R=\left[\left(x+x^{\prime}\right)^{2}+\left(y+y^{\prime}\right)^{2}\right]^{1 / 2} \&$

$x^{\prime}=a \cos \phi, y^{\prime}=a \sin \phi \cos \delta, z^{\prime}=-a \sin \phi \sin \delta$ 


\section{Analysis}

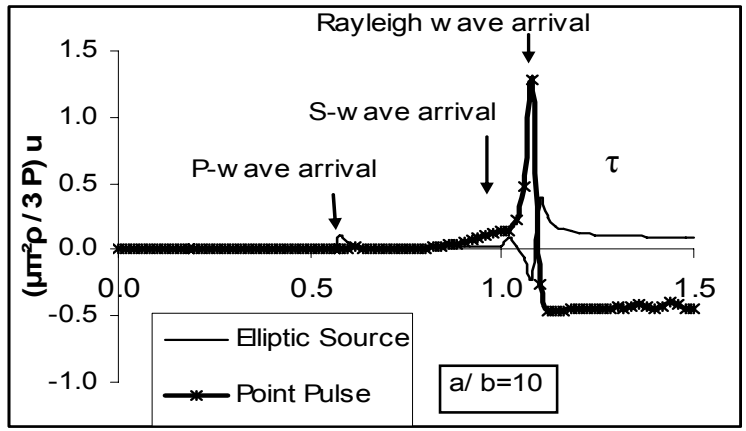

Figure 3(a)

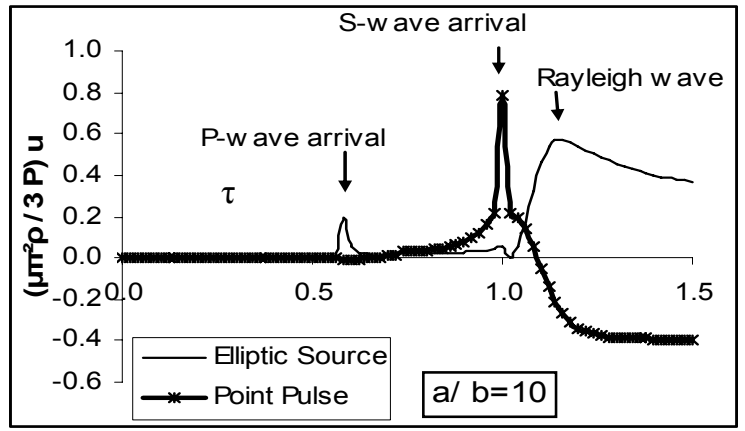

Figure 3(b)

Comparison of dimensionless vertical surface displacements at (30,40,-1) (Figure 3(a)) and (3, 4,-1) (Figure 3(b)) on the surface of an elastic half-space due to a buried point pulse and a horizontal elliptic source $\left(\delta=0^{\circ}\right)$ of same magnitude.

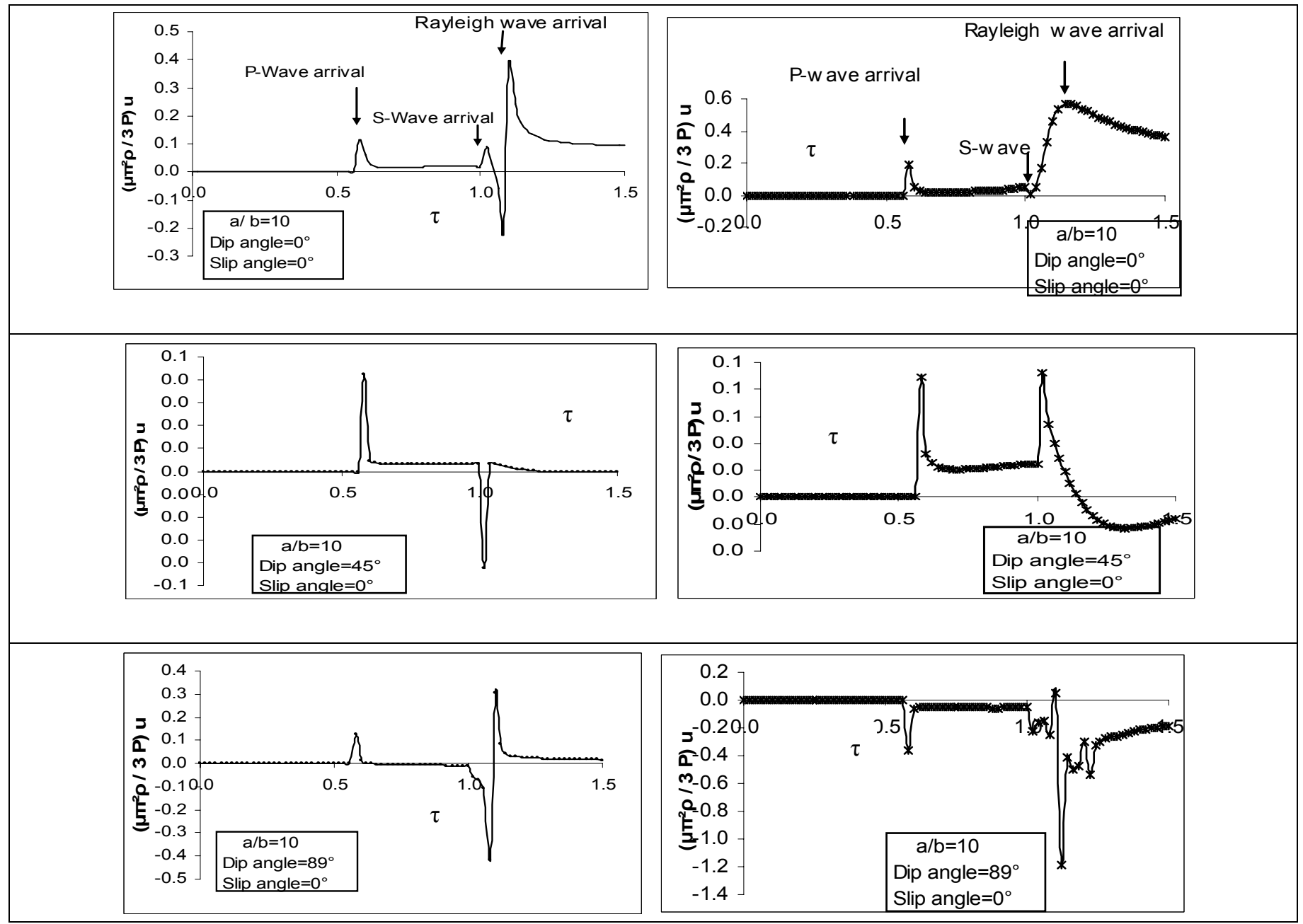

Figure 4(a)

Figure 4(b)

Comparison of dimensionless vertical surface displacements at (30,40,-1) (Figure 4(a)) and (3, 4,-1) (Figure 4(b)) on the surface of an elastic half-space due to a buried elliptic source at different dip angles $(\delta)$. 


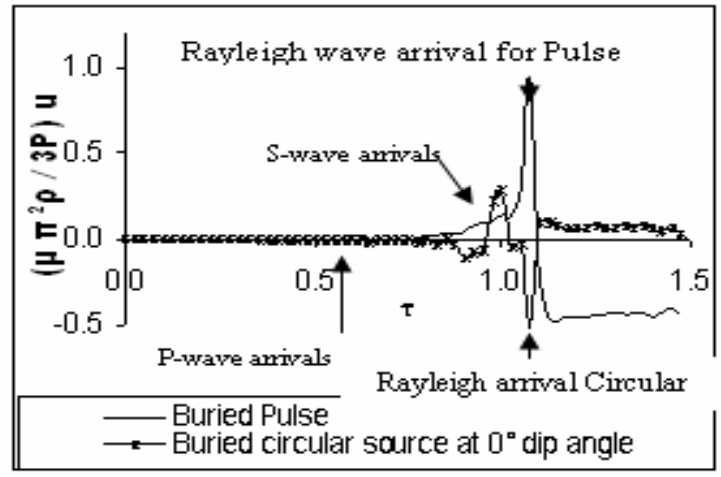

Figure 5(a)

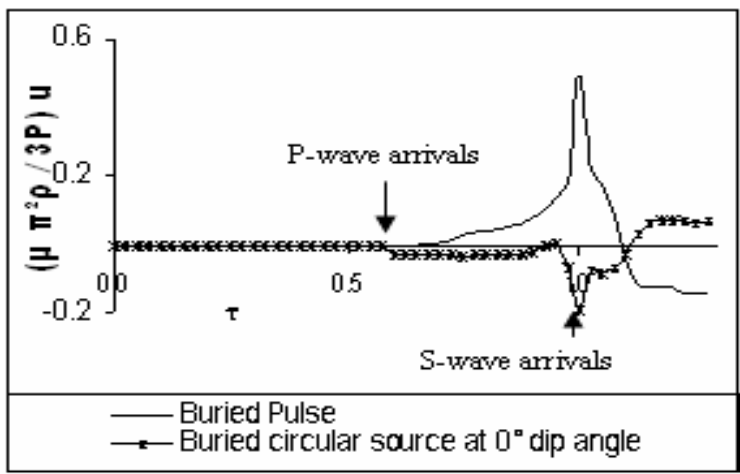

Figure 5(b)

Comparison of dimensionless vertical surface displacements at $(30,40,-1)$ (Figure 5(a)) and $(3,4,-1)$ (Figure 5(b)) on the surface of an elastic half-space due to a buried Pulse and a horizontal circular source $\left(\delta=0^{\circ}\right)$ of same magnitude

\section{Conclusion}

The transformed vertical surface displacement field in (2.4) for the normal impulsive pressure on an elliptic area along an inclined plane agrees with that of Pekeris and Lifson (1957) in an uniform elastic half-space (Lame's constants $\lambda=\mu$ ) for a buried pulse and can be derived after dividing the equation (2.4) by $\pi \mathrm{ab}$ for $\mathrm{a}=\mathrm{b}$ and making limit a tends to zero for $\delta=0^{\circ}$. The Bessel function appearing here to be substituted by its proper integral representation. The dimensionless vertical surface displacement field as presented in section 4 has an additional integral over $-\pi$ to $\pi$ which arises due to the finite nature of the elliptic source. Results of Roy (1975) for a pulse in an elastic half-space also agree with our present results in section 4.

$I_{j}\left(q_{j}^{+}, \omega_{j}\right)$ in the final expression includes both the effects of the source distribution and the elastic medium. The center of the elliptic source is situated at a depth " $\mathrm{h}$ " below the surface of the uniform elastic half-space. We have computed various surface responses at two arbitrarily chosen representation points, called near [i.e., $(3,4,-1)] \&$ away [i.e., $(30,40,-1)]$ points, on the surface due the normal impulsive pressure along an inclined fault plane for various dip angles of the fault plane. The figures $3(\mathrm{a}) \& 3$ (b) show the comparison of the dimensionless vertical surface displacements due to a buried horizontal $\left(\delta=0^{\circ}\right)$ elliptic source along with a pulse of same magnitude at two representation points for $a / b=10$. The results obtained for the pulse are in accordance with that of Pekeris and Lifson (1957). The initial direction of the vertical displacement at the P-wave arrival for the away point is same as that of point pulse $\left(\delta=0^{\circ}\right)$. But for the near point they are opposite for the ratio of semi-axes $\mathrm{a} / \mathrm{b}=10 \& \delta=0^{\circ}$. The Rayleigh wave arrivals for both the sources are marked prominently in opposite directions at the away point for $\mathrm{a} / \mathrm{b}=10$ (Figure 3(a)) and at the near point it is marked in positive direction for the elliptic source only (Figure 3(b)). The dimensionless vertical surface displacement at the Rayleigh wave arrival for the elliptic source is blunt in compared to that of point pulse (Figure 3(a,b)). The S-wave arrival is only marked prominently for the point pulse at the near point for $\delta=0^{\circ}$ and $\mathrm{a} / \mathrm{b}=10$ (Figure $3(\mathrm{~b})$ ). Similar type of analysis can be made for a circular source (Figure $5(\mathrm{a}, \mathrm{b})$ ). The Rayleigh wave arrival for a pulse is marked by an infinite discontinuity while that of circular source is blunt (Figure 5(a)) for the away point only. The S-wave arrivals at the near point are marked prominently in the opposite directions for both the sources (Figure 5(b)).

Figures 4(a) \& 4(b) give us a comparison of the dimensionless vertical surface displacements at the away and near points (viz, $(30,40,-1) \&(3,4,-1))$ for the buried elliptic source at different dip angles. The amplitude at the P-wave arrival gradually decreases with the gradual increase in inclination of the fault plane for the away point (Figure 4(a)). But for the near point it is in opposite direction at $\delta=89^{\circ}$ in compared to that for other values of $\delta$. The S-wave arrivals at $\delta=45^{\circ}$ are prominently marked for both away and near points but in opposite directions. The direction of the dimensionless vertical surface displacement at the Rayleigh wave arrival for the near point at $\delta=0^{\circ} \& 89^{\circ}$ are opposite with increasing amplitude. Similar type of analysis can be made for other points. The dimensionless surface displacements for the other realistic fault geometry can be obtained by the method presented here. 


\section{Appendix}

The expressions of different terms used in section 4 are as follows

$$
\begin{aligned}
& N^{\prime}{ }_{P}(q, \omega)=\frac{i}{2 \pi}\left[\frac{P a\left(\eta \sin \delta+i \zeta_{P} \cos \delta\right)\left\{i\left(2 \xi^{2}+2 \eta^{2}+\left(\frac{\alpha^{2}}{\beta^{2}}\right)\right\}\right.}{2 \pi \mu . F(\xi, \eta)\left(\frac{a^{2}}{b^{2}} \xi^{\prime 2}+\eta_{p}^{\prime 2}\right)}\right] \frac{\left(\frac{a}{b} \xi^{\prime} \cos \phi+\eta_{p}^{\prime} \sin \phi\right)\left(\xi^{2}+\eta^{2}+1\right)^{1 / 2}}{\left(q^{2}+\omega^{2}+1\right)^{1 / 2}} \\
& N^{\prime}{ }_{S V}(q, \omega)=-\frac{i}{2 \pi}\left[\frac{P a\left(\eta \sin \delta \zeta_{S}+i\left(\xi^{2}+\eta^{2}\right) \cos \delta\right)\left\{2 i \zeta_{P}\right\}}{2 \pi \mu . F(\xi, \eta)\left(\frac{a^{2}}{b^{2}} \xi^{\prime 2}+\eta_{s}^{\prime 2}\right)}\right] \frac{\left(\frac{a}{b} \xi^{\prime} \cos \phi+\eta_{s}^{\prime} \sin \phi\right)\left(\xi^{2}+\eta^{2}+\frac{\alpha^{2}}{\beta^{2}}\right)^{1 / 2}}{\left(q^{2}+\omega^{2}+\frac{\alpha^{2}}{\beta^{2}}\right)^{1 / 2}}
\end{aligned}
$$

\section{References}

Gakenheimer D.C. and Miklowitz, J., 1969. Transient Excitation of an Elastic Half Space by a Point Load Traveling on the Surface, Journal of Applied Mechanics, Vol.36, pp. 505-515.

Gridin D., 1999. On the Radiation of Ultrasound into an Isotropic Elastic Half-space via Wave Front expansions of the Impulse Response, Journal of Acoustical Society of America, Vol.105, No.5, pp. 2565-2573.

Mitra M., 1964.Disturbance Produced in an Elastic Half Space by Impulsive Normal Pressure, Proceedings of the Cambridge Philosophical Society, Vol.60, pp.683-696.

Pekeris C.L.and Lifson,H.,1957. Motion on the Surface of a Uniform Elastic Half space Produced by a Buried Pulse, Journal of Acoustical Society of America, Vol.29, pp.1233-1238.

Roy A., 1979.On Dislocations and Extended Sources in an Elastic Half-space, Indian Journal of Pure and Applied Mathematics,Vol.10, No.1, pp.100 - 111.

Roy A., 1975. Pulse Generation in an Elastic Half space by Normal Pressure, International Journal of Engineering Sciences, Vol.13, pp. 641- 651.

Roy A., 1981. Response of an Elastic Half-space to Normal Pressure over an Elliptic Area, International Journal of Engineering Sciences, Vol.19, pp.129-136.

Savage J.S.,1966. Radiation from a Realistic Model of Faulting, Bulletin of Seismological Society of America, Vol. 56, pp.577592.

Tupholme G.E., 1970. Pulse Generation in an Elastic Half space by the Uniform Normal Loading of a Circular Surface Segment, International Journal of Engineering Sciences, Vol. 8, pp.617-639.

Zeng S. and Liang R., 2002. A Fundamental Solution of a multilayered Half-space due to an Impulsive Ring Source, Solid Dynamics and Earthquake Engineering, Vol.22, No.7, pp.541-550.

\section{Biographical notes}

Arabinda Roy received Ph.D. from Calcutta University, India. He is a retired Professor in the Department of Applied Mathematics, Calcutta University, Kolkata, India. He has more than 35 years of experience in research and teaching. He has published many research papers in referred international and national journals.

Ajit De is an Assistant Professor in the Department of Mathematics, Siliguri College, Siliguri, India. He has received M.Phil. from Calcutta University, India. He has more than 8 years of experience in teaching and research. His current topic of research is seismology. His one paper currently has been accepted in an international journal. He has recently communicated one paper in a referred journal.

Received September 2010

Accepted June 2011

Final acceptance in revised form June 2011 